\title{
Der Ursprung der Virgilsage.
}

Der fast einstimmige Beifall, welchen D. Comparettis eingehende Behandlung der mittelalterlichen Virgiltradition ${ }^{1}$ gefunden hat ${ }^{2}$, könnte eine neue Arbeit über diesen Gegenstand von vornherein als unberechtigt erscheinen lassen. Es ist ebensowenig meine Absicht, Comparettis Buche Verdienste abzusprechen, durch die es in der That epochemachend dasteht, als hier zweifellose Ergebnisse noch einmal zu entwickeln. Mir scheint indessen die Untersuchung eben doch zu einem nur vorläufigen Abschluss gebracht. Durch die nachstehenden Bemerkungen möchte ich auf einige entscheidende Punkte aufmerksam machen, die, wie ich glaube, noch nicht zur endgültigen Feststellung gelangt sind. Erweisen sich die hier erhobenen Zweifel an der Richtigkeit von Comparettis Resultaten als unbegründet, so werden sie dazu wenigstens dienen, die letztere in ein um so helleres Licht $\mathrm{zu}$ stellen.

Es dauerte lange, bis der Virgilsage, nachdem sie in den Volksbüchern in ihre letzte Phase getreten war, eine wissenschaftliche Beachtung zu Theil ward. Langsam überzeugte man sich, dass der Virgil der Sage kein anderer sei als der allbekannte der Geschichte. Der Sänger der Aeneide ein abenteuerlicher Zauberer! Woher diese erstaunliche Verwandlung? Das war die Frage, die nun an die Kritik herantrat.

Freilich wagte man sich vorerst noch nicht an die Lösung des Räthsels. Die ersten Arbeiten über den mittelalterlichen Virgil, wie die von Siebenhaar, Michel und Schubbe, gaben nur einstweilen das nöthigste geschichtliche Material. Hierauf versuchte Du Méril ${ }^{3}$ mit reicheren Mitteln, aber auf falschem Wege, den Ursprung der Sage nachzuweisen. Ueber dem „Ergänzen einer Relation durch die andere und dem Ausscheiden des willkürlich Scheinenden" vergisst $\mathrm{Du}$ Méril, dass seine Aufgabe vor allem die historische, nicht psychologische Erklärung der Sage war. Die Ansicht, dass die von den Quellen gebotenen traditionellen Data im Grunde nichts seien

1 Virgilio nel medio evo. Livorno 1872. 2 Bde.

2 Vgl. jedoch E. Stengel, Fenaer Litteraturzeitung 1874. S. 72 Sp. 2.: "Comparettis Annahme einer Trennung von Volkssage und litterarischem Mythus ist ebenso unnöthig wie willkürlich." téraires. Paris 1850.

${ }^{3}$ De Virgile lenchanteur. In seinen Mélanges archéologiques et litZeitschr. f. rom: Ph. 1. 
als „Superstitionen, die sich das Volk zur Stütze seiner vorgefassten Mcinungen ersinnt", hat zu der schlimmen Consequenz geführt, dass Du Méril nicht mit jenen überlieferten Notizen, sondern deren muthmasslichen Grundideen zu operiren versucht hat. Dass es ihm hicrbei auf chronologische Ordnung und kritischen Werth der Quellen nicht sonderlich ankam, ist natürlich. Immerhin gewinnt man aus Text und Belegen der Du Mérilschen Arbeit schon den Eindruck, dass die ausgebildete Sage nur eine Entwicklung der in der Litteratur früherer Perioden verbreiteten Ideen über den Dichter ist. Nun scheinen aber gerade die ältesten Zeugen eigentlich legendenhafter Züge im Gegensatz zu einer allmählichen litterarischen Entwicklung eine durch locale Einflüsse bedingte Entstehung der ältesten Sage zu verbürgen. Du Méril, dem die Wichtigkeit dieser ersten Lebenszeichen der wirklichen Sage vollkommen entging, wurde der neuen Schwierigkeit nicht gewahr. Ebensowenig Zappert 1 und Genthe ${ }^{2}$. Des Ersteren Schrift enthält eine nicht uninteressante Sammlung virgilianischer Reminiscenzen bei Schriftstellern des Mittelalters, bietet aber keinen Beitrag zur Kritik der Sage. Genthe will allerdings zeigen, „dass die Vorstellung von Virgilius als einem grossen Meister in den Geheimnissen der Natur, als einem Necromanten, keine willkürliche Annahmen einzelner Schriftsteller waren, sondern dass dieser wirkliche Volkssagen zu Grunde lagen“, gibt aber zu diesem Zwecke nur eine lange Liste von Autoren, die der Zauberei Virgils Erwähnung thun.

Roth glaubte daher in seinem im Jahre $185^{8}$ erschienenen Aufsatze ${ }^{3}$ noch einmal die Fragen aufwerfen zu müssen, „ob der Zauberer mit dem Dichter nachweislich zusammenhange, wann und wo das Mährchen entstanden, wie seine Verbreitung vor sich gegangen und aus welchen Ursachen seine Entstehung zu erklären sei.“ Er gelangte mit Zugrundelegung der ältesten Berichte zu dem Resultate, ,dass nur die neapolitanische Sage ächte Volkssage genannt $\mathrm{zu}$ werden verdient, die römische hingegen und die mantuanische nur eine litterarische und zwar wesentlich französische Entstehung hat, und darum [?] jener gegenüber eine unzusammenhangende, willkürliche und unberechtigte heissen muss."

Abgesehen von der Zuverlässigkeit dieser Ergebnisse, auf die wir uns sogleich etwas näher einzulassen haben, blieb nach dieser ersten wissenschaftlich bedeutenden Arbeit über den Zauberer Virgilius eine eingehende Darlegung der von unserem Dichter im Mittelalter behaupteten Stellung ein vielversprechender Vorwurf. Eine Lösung dieser Aufgabe liegt in dem Werke Comparettis vor.

Mit Recht betrachtet es Comparetti als erstes Erforderniss, die Fäden nachzuweisen, durch welche die Virgilsage mit dem Leben

1 Virgils Fortleben im Mittelalter. Wien (Berichte a. Ak. d. W.) 1851 .

2 Leben und Fortleben des P. Virgilius Maro als Dichter und Zauberer. Leipzig I857.

${ }^{3}$ Ueber den Zauberer Virgilius. In Pfeiffers Germania, Bd. IV. 
des Dichters verknüpft ist. $\mathrm{Zu}$ diesem Ende gibt der Verfasser zuerst eine erschöpfende, in solcher Ausdehnung vielleicht etwas aus dem Rahmen des Buchs heraustretende Darstellung von Virgils Erfolg und Einfluss von seinem Bekanntwerden bis zum Anfang des Mittelalters. Hiernach ward der Aeneide, die in jeder Beziehung dem nationalen Bedürfniss entsprach, von Seiten des römischen Publicums freilich nicht eine vollkommene Würdigung, wohl aber extremer Enthusiasmus (und daneben Sarcasmus) zu Theil. Der Dichter erlangte die grösste Popularität in der Gesellschaft wie in der Schule, bei den Rhetoren wie bei den Poeten. Für Virgils fortdauerndes Ansehn in der Zeit des Verfalls bürgen im Besonderen noch die Centonen und die Commentare; Macrob erklärt ihn für erfahren in jedem Wissenszweige und frei von Irrthum. Als Autorität für die Grammatik behauptet er sich gegen den feindlichen Andrang des Germanen - und Christenthums, bis im I 2. Jh. die Scholastik auch auf diesem Gebiete Umwälzungen hervorrief.

Von den älteren Bestandtheilen der Virgilbiographien, auf welche Comparetti erst nach der Untersuchung über Virgils Stellung im Mittelalter im Allgemeinen zu reden kommt, ist allerdings nur die Erzählung von dem Pappelzweig für uns von Interesse. Gegenüber Comparettis Geneigtheit, die Notiz für theilweise glaubwürdig zu halten, könnte man meinen, dass bei der virga populea der Name Virgilius nicht ganz unbetheiligt sei. Doch sind ja, wie auch Comparetti bemerkt, Anekdoten ähnlicher Art im Alterthum etwas zu Gewöhnliches, als dass die in Rede stehende des Dichters Ansehn ein besonderes Colorit verleihen konnte. - Schwerer lässt sich indess mit den interpolirten Fabeln der Vita-Handschriften aus dem I4. Jh. fertig werden.

$\mathrm{Da}$ die ältesten der uns überhaupt überlieferten Virgilsagen fast ohne Ausnahme in ausdrücklicher Beziehung zu Neapel stehen und nachweislich ins I 2. Jh. hinaufreichen, so hat Roth im Zusammenhang mit seinen sonstigen Ergebnissen, die Interpolationen der Biographie einem neapolitanischen Gelehrten jener Zeit geglaubt zuschreiben zu sollen. Diese Ansicht steht und fällt mit Roths Gesammtbeurtheilung unsrer Tradition. Was Comparetti dagegen geltend macht, lässt sich auf folgende Gründe reduciren: I) Obwohl nach Zahl und Beschaffenheit in den einzelnen Handschriften nicht verschieden, sind die Einschiebsel offenbar nicht das Werk eines Mannes und einer Zeit. 2) Der Inhalt eines derselben findet sich schon bei Servius, bei Cassiodor, bei Aldhelm. 3) Ihre Abfassung durch einen neapolitanischen Gelehrten des 12. Jh. setzt für Letzteren eine zu hohe Bildungsstufe voraus. - Das zweite Argument ist schlagend. Kannte man die Fabeln zum Theil schon Jahrhunderte früher in gelehrten Kreisen ausserhalb Neapels, ja ausserhalb Italiens, so fehlt jeder Anlass, sie sich als Localsagen von einem gelehrten Neapolitaner aufgezeichnet zu denken. Im Uebrigen wird man sich bei Comparettis Vermuthungen einstweilen beruhigen müssen: irgend ein Grammatiker hat die Erzählungen der Vita des 
Donat einverleibt; mögen diese nun vor dem 6. Jh. einer Biographie angehört oder vereinzelte Anekdoten gebildet haben.

Augenscheinlich aber ist Roths Hypothese unter dem Eindrucke jenes oben berührten Widerspruches gelehrten und localen Characters der Sage entstanden. Roth räumt der localen Entstehung den Vorrang'ein, ohne gelehrten Ursprung negiren zu können, und personificirt die Versöhnung der Gegensätze zu einem neapolitanischen Gelehrten.

Wie zieht sich nun Comparetti aus diesem Dilemma? War Roth, die innere Einheit und Untheilbarkeit der Sage wohl erkennend, an der äusseren Verbindung der Gegensätze gescheitert, so wird von Comparetti diese Klippe dadurch vermieden, dass er gelehrte und populäre Tradition gänzlich auseinanderreisst. Er erklärt es ausdrücklich für einen Irrthum, wenn man die Interpolationen der Vita des Donat und gewisse andere Data mittelalterlicher Biographien als der Legende von Virgil dem Zauberer entsprungen betrachte. Dieser Irrthum beruhe auf der Vermengung zweier Dinge, durchaus verschieden durch Beschaffenheit, Zeitalter und Ursprung, der Volkssagen nämlich und der Gelehrtensagen. Bemerkenswerth ist es, dass Comparetti gleich folgen lässt: „Fra queste due categorie di prodotti favolosi ¿’è invero una comunanza di base, poichè ambedue partono da una idea esagerata della sapienza virgiliana (Comp. I, I $86 \mathrm{f}$. I 90 ).

Stellen wir uns nun die Gründe zusammen, die jene Trennung rechtfertigen sollen. Es sind zunächst innere: I) Gröbere Auffassung von Virgils Weisheit in der Volkssage. 2) Verschiedenes Feld der Thätigkeit Virgils: in der Volkssage verliert Virgil ganz den Charakter des Dichters; in der Gelehrtensage bleibt er stets Dichter. 3) Die Gelehrtensage hat genügende Gründe und Ursachen in den vorausgegangnen historisch-psychologischen Erscheinungen, welch letztere aber nicht ausreichen würden den Ursprung der Volkssagen zu erklären.

Das unter I) Behauptete ist an und für sich nicht in Abrede zu stellen. Beweist denn aber diese Thatsache etwas für einen grundverschiedenen Ursprung der volksthümlichen Erscheinungsform der Sage?

Gibt Comparetti zweitens vor, Virgil behalte in der Gelehrtensage seinen Charakter als Dichter, verliere ihn aber in der Volkstradition, so genügt einstweilen der Verweis einerseits auf die eingestandnermassen litterarisch entstandnen interpolirten Vita-Anekdoten, andrerseits auf die für volksthümlich erklärten Berichte der Cronica di Partenope, um dieses Argument gegen einen gemeinsamen Ursprung sämmtlicher Virgilsagen aus dem Wege zu räumen.

Was nun endlich den dritten Einwand betrifft, so gibt uns Comparetti selbst das vollständige Material an die Hand zu folgenden Beweisen für die Möglichkeit der litterarischen Entstehung der Sage.

I. Die Richtung und der seitherige Fortschritt in der Entwicklung der litterarischen Tradition. - Einmal galt Virgil in gelehrten Kreisen längst für einen übernatürlich begabten Meister in allen 
Disciplinen (vgl. Comp. I, 85). Auf der andern Seite stand der Dichter schon in den historischen Angaben der Vita in Beziehung zu der Stadt Neapel. Dass er - nach Alexander von Telesa „Neapolis civitatis simulque Calabriae dominatus caducam ab eo [Octavian] recepit retributionem", ist hiernach ohne Einwirkung einer für sich ja noch gar nicht bezeugten neapolitanischen Ortssage aus der geschichtlichen Tradition zu erklären, die schon seither stets wachsende Gunstbezeugungen des Kaisers zu berichten hatte (vgl. Comp. I, I 96 ff.).

2. Missverständniss und Fälschung der überlieferten Thatsachen durch die zunehmende Unwissenheit in gelehrten Kreisen. Welche Versehen, welche Phantastereien waren denn unmöglich bei Leuten, die, wie Smaragd, Eunuchus comoedia und Orestis tragoedia für Autorennamen ansahen; die, wie Remigius von Auxerre, poema mit positio, emblema mit habundantia übersetzen, oder die efficiam durch effigiem, imaginem, und provincia durch celeramente erklären, Quo te Moeri pedes als Quot Emori pedes lesen und darin eine Anspielung auf ein schnelles sarazenisches Pferd Emoris finden, elenco für mit Titel gleichbedeutend halten und vom griechischen „eleos" ableiten, weil, wie die Sonne die Welt, so der Titel das Buch erleuchte (Comp. I, I69 ff.), und die in der Geschichte nicht mehr leisten als in der Etymologie (vgl. Comp. I, I 72)?

3. Die Mitwirkung der auf Abenteuerliches und Wunderbares gerichteten Vulgärpoesie. - Denn, nach Comparetti selbst: gerade im 12. Jh. ", ha luogo in modo efficace il connubio intimo, benchè alla prima quasi paradossale, fra la poesia romantica e cavalleresca di origine affatto popolare, e la cultura, la tradizione e il sapere" (Comp. I, 248 ; vgl. II, 4 etc.).

4. Die Thatsache, dass wenigstens ein gelehrter Autor die Zauberkunst Virgils auf rein litterarischem Wege entstehen lässt („Et fuit magnus magicus ut patet ex illa ecloga Pastorum musam" etc.; Comp. I, I 95 f.), wobei es gleichgültig ist, ob ihm gerade die neapolitanische Zaubersage bekannt war oder nicht. Auch der Dolopathos zeigt sich unabhängig, nicht von volksthümlichen Sagenkreisen, wohl aber, was Comparetti besonders hervorhebt, von localisirten Legenden. Gleichwohl erscheint darin Virgil als "un savio versato in tutte le discipline che allora constituivano la scienza, compresa l'astrologia più mirabile e più fantastica fra tutte" (Comp. II, I 4). Gibt nun Comparetti ausdrücklich $\mathrm{zu}$, dass der Zauberer eigentlich ein ,accrescitivo del gran sapiente, in certo senso anche un peggiorativo, come caratteristica morale" ist, so hat die wiederholte Versicherung, dass die Idee des Zauberers ihren Ursprung ausserhalb der Schule habe und aus dem schulmässigen Virgiltypus nicht erklärlich sei, allen Boden verloren, und es bedürfte der trotz aller Clauseln vollständigen Selbstwiderlegung nicht: "Che l'antico savio si cambi in mago è fatto di cui rari sono gli esempi ${ }^{1}$, e quando accade ha

1 Vgl. übrigens Du Méril, l. c. S. 448; Liebrecht, Des Gervasius von Tilbury Otia Imperialia in einer Auswahl. Hannover I856. S. XVIII. 
luogo per puro cambio di nome $e$ in modo momentaneo; non v'ha antico che arrini mai a quel largo e completo ciclo di leggenda biografica che ebbe il Virgilio mago" (Comp. II, I 5).

Auf welche historisch-psychologischen Erscheinungen gründet nun seinerseits Comparetti die von ihm angenommene populäre und locale Entstehung der Sage? Fr erkennt zwei Elemente in der altesten Gestalt der neapolitanischen Tradition: I) den von der Vorstellung einer besonderen Zuneigung, die der Dichter zu der Stadt gehabt habe, begleiteten Namen Virgils; 2) den Glauben an einige ihm zugeschriebene öffentliche Talismane (Comp. II, 55).

Weshalb hätte aber das Volk von Neapel an jene besondere Zuneigung glauben oder, was Comparetti ebenfalls wahrscheinlich findet, in Virgil den Beschützer der Stadt erblicken sollen, und zwar noch elf Jahrhunderte nach seinem Tode? Ausser dem der Grabstätte des Dichters bewahrten Andenken, erklärt Comparetti, hätte unzweifelhaft Virgils Name durch einige Landgüter, die er dort besessen, erhalten werden müssen (II, 49). Nun findet sich, wie Comparetti zeigt, allerdings ein mons Virgilii bei einigen, und zwar kirchlichen Schriftstellern; gewöhnlich und, was nicht zu übersehen ist, auch bei Gervas, heisst er jedoch mons Virginum, heutzutage Monte Vergine. Diese Argumente sind so hinfällig, und der Zusammenhang mit der gelehrten Tradition so deutlich, dass Comparetti gleichsam unwillkürlich das Zugeständniss macht: ,il popolo adunque non faceva altro a Napoli se non trarre conseguenze materiali dal concetto che $i$ letterati d'allora si formavano di Virgilio" (II, 42).

Wenn nun aber die Grundidee der neapolitanischen Sagen nicht dem Volke von Neapel entstammt, welche Wahrscheinlichkeit hat es für sich, dass die blosse Existenz einiger Kunstgegenstände dieses hätte veranlassen sollen, dem Virgil die nach Comparetti von der des litterarischen Virgil so sehr abweichende Rolle des Zauberers beizulegen?

Doch wir haben es bisher nur mit den inneren Gründen für den doppelten Ursprung der Sage zu thun gehabt. Erschienen uns dieselben nicht geeignet, die Nothwendigkeit der Annahme eines solchen erkennen zu lassen, so wird vielleicht seine Thatsächlichkeit durch äussere Belege hinreichend sicher gestellt? In der That sucht ja Comparetti, was vor ihm schon Roth und alle Andern als unzweifelhafte Thatsache angenommen, durch positive Beweisführung ausser Frage zu stellen, dass - wenn wir die von Comparetti vorausgeschickten Behauptungen zu ordnen versuchen - die neapolitanische Virgillegende eine durchaus volksthümliche nicht-litterarische Localsage sei, die sich gründe auf Reminiscenzen an Virgils Aufenthalt in Neapel, auf das Vorhandensein und die Berühmtheit seines Grabes, sowie auf neapolitanische Oertlichkeiten, Bildnisse und Denkmäler (denẹn eben Virgil eine Zaubermacht verliehen haben sollte). Ist nun offenbar der Beweis für diese Punkte von litterarischen Zeugnissen zu erwarten, so dürfte es von vornherein Bedenken erregen, dass solche sich ,assai piü e prima fuori d'Italia che in 
Italia" finden, dass die Tradition "contemporaneamente in opere volgari e romantiche ed in opere latine di natura dotta" übergeht, und dass sie auftritt gerade „dal XII secolo in poi, ossia dall'origine della poesia e prosa romanzesca di proprio nome" (Comp. II, I $8 \mathrm{f}$.).

Roth wie Comparetti stützen ihre neapolitanische Volkssage vor allem auf die Angaben zweier Autoren, welche ihre - vorgebliche - Augenzeugenschaft jedesfalls mehr empfiehlt als ihre gesellschaftliche Stellung, die Comparetti, oder ihre Nationalität und Bildung, die Roth hervorhebt. Ehe wir Konrad von Querfurt und Gervas von Tilbury zur Zeugnissablage zulassen, erscheint es geboten, sich über ihre allgemeine Glaubwürdigkeit ein Urtheil $\mathrm{zu}$ bilden.

Was zuerst den Kanzler Heinrichs VI. und dessen Vertreter in Neapel und Sicilien, späteren Bischof von Hildesheim, Konrad von Querfurt betrifft, so muss Comparetti selbst eingestehen, dass derselbe manchmal glaubte, ,vedere altro $e$ più di quello che realmente vedesse:“ - „è impossibile spiegare altrimenti certi grossi svarioni del bravo cancelliere messi giù con una serietà da far disperare" (II, 2 I f.). Auch Roth gibt zu, „dass es ein Lächeln erregen muss, dass auch die Berge Olympus und Parnassus, die Quelle des Pegasus, die Insel des Achilleus, der Palast der Helena, der Stall des Minotaurus und andre griechische Oertlichkeiten ihm entweder durch eine lebendige Phantasie vorgezaubert oder von schlauen Ciceroni gewiesen worden sind.“ Führt die letztere Auffassung zu einer ebenso stupenden Weisheit des Cicerone als Albernheit des Reisenden, so müssen bei Annahme auch der lebendigsten Phantasie die Berichte eines solchen Gewährmannes nicht sowohl ein Lächeln als erhebliche Zweifel an deren Zuverlässigkeit hervorrufen; und wenn Comparetti mit Recht meint, beim Gedanken an Maundeville könnten solche Erzählungen nicht auffallend erscheinen, so darf er für sie eben auch keine grössere Autorität in Anspruch nehmen als für die des englischen Reisedichters.

Ob Konrads Bericht (I I 94) von den Adressaten durchaus für baare Münze genommen worden sei, wie Leibnitz annimmt, will ich dahin gestellt sein lassen. $\mathrm{Zu}$ der Behauptung, dass Arnold von Lübeck den Brief in seine Chronica Slavorum 1 eingefügt habe, "tamquam auro includeret gemmam", berechtigt dessen Bemerkung "Nec piguit ad aedificationem aliorum hic ponere epistolam Conradi Cancellarii, quam scripsit nobis de statu Apuliae et de operibus vel artibus Virgilii“ jedenfalls nicht; vielleicht klingt aus den Worten „ad aedificationem aliorum" eine leise Ironie. Uebrigens scheint mir aus der Art, wie die „Werke oder Künste Virgils" erwähnt werden, fast hervorzugehen, dass dieselben dem Schreiber (und nach dessen Voraussetzung auch wohl den Lesern) der Chronik bekannt waren. Dass wenigstens die Empfänger des Briefes von den „Werken Virgils“ schon etwas gehört hatten, lehrt eine Stelle des Briefes selbst, nach der wir meines Erachtens den ganzen Inhalt desselben zu beurtheilen haben. Uns ist es zu Theil geworden, schreibt Konrad im Eingang,

${ }^{1}$ Bei Leibnitz, Scriptores rerum brunsvicensium II, 695-8. 
"ut ea quae olim apud vos in scholis positi in aenigmate quasi in speculo aure tenus percepimus, facie ad faciem oculata nunc fide cognoverimus;" und weiter unten - gewiss ebenso zu verstehen ..., ,ut ad ea videnda quae auditis vestrum accendamus desiderium, ut ea quat nunc demissa tantum per aurem vobis sunt dubia, fide plena constent, et pateant occulta." Der Kanzler hat also einfach die Absicht, seinen alten Freunden mit der auf dem classischen Boden Italiens selbstgewonnenen Bestätigung bekannter Schultraditionen zu imponiren, wobei er es nach dem Brauch der Zeit mit der Wahrheit seiner Angaben nicht sehr genau nimmt. Gelehrte Reminiscenzen bilden die Citate aus Virgil und Ovid. Vielleicht dürfte man in der Zusammenstellung der Bäder Virgils mit dem Bade der Sibylle eine Einwirkung der Idee von Virgils Prophetismus erblicken. Jedenfalls liegt weder eine Nöthigung vor die Mittheilungen über die artes Virgilii anders aufzufassen als den Rest des Briefes ${ }^{1}$, noch enthalten diese Notizen eine Rechtfertigung der Angabe von Roth und Comparetti, Konrad beabsichtigte eine "objective Berichterstattung“ oder gar, er habe seine Legenden ,aus dem Munde des Volkes von Neapel“. Selbst wenn Konrad solche Behauptungen aufstellte, so nöthigte uns nichts daran zu glauben. Aber er sagt ganz einfach, dass die Wunderdinge da sind oder da waren, und nur einmal, dass er gesehen habe, was er berichte, niemals jedoch, er habe „idee allora proprie del popolo napoletano" gesammelt.

Wir gehen über zur Charakteristik des zweiten Hauptzeugen, Gervasius von Tilbury. Auch zur Empfehlung des Professors an der Universität Bologna und Marschalls von Arles lässt sich wenig sagen. Des Gervasius Werk, die I 2 I 2 geschriebenen Otia Imperialia, enthält nach Comparetti "notizie d'ogni sorta e assurdità d'ogni calibro." Wenn aber Roth angibt, willkürliche Erdichtungen und Lügen seien Gervasius seines Wissens nicht Schuld gegeben worden, so war dies allerdings sowohl von Seiten Liebrechts als schon durch Leibnitz geschehen. Ersterer bemerkt, „dass er einen beträchtlichen Theil seiner Arbeit und daher auch die darin vorkommenden Citate der Historia scholastica des Petrus Comestor meist wörtlich entliehen hat, ohne denselben auch nur ein einziges Mal wörtlich anzuführen. Ja er nimmt sogar nicht Anstand,“ fährt Liebrecht fort, „,ausdrücklich sich selbst zuzuschreiben, was er doch nur aus Comestor hat;" 2 und Leibnitz sagt in seiner, wie es scheint, zu wenig berücksichtigten, freilich etwas weitschweifigen Einleitung: Caeterum Gervasium .... mox ubi sacra et remota a suis temporibus attingit, mirificentissimum

${ }^{1}$ Sie sind folgendermassen eingefuhrt: - Praeteriimus deinde fovianam civitatem quae quasi fovis natio nuncupatur eo quod ibi Fupiter natus fuerit. Vidimus etiam .... (es folgen einige der Virgilsagen). In principio ergo Siciliae vidimus domum Daedali“ ... Nach einigen andern Erzählungen kommt Konrad auf Neapel zurück: "Caeterum ad mentem reducimus quod apud Neapolin est quaedam porta." etc.

${ }^{2}$ Liebrecht, l. c. S. IX. Mit der Bemerkung: „Von dem Vorwurfe der absichtlichen Lügenhaftigkeit, den ihm Leibnitz macht, möchte ich ihn freisprechen" (Liebr. l. c. S. X) widerspricht Liebrecht sich selbst. 
et superstitiosissimum fabulatorem fatendum est. Sed neque prorsus eum a mentiendi libidine absolverim. Quaedam enim velut testis oculatus narrat, quae nemini mentis compoti hodie facile persuaseris." Die Lectüre der Otia setzt die Richtigkeit dieses Urtheils ausser Zweifel. 1 Gervasius verdient hiernach nicht mehr Glauben als sein Vorgänger Konrad. Erweisen lässt sich allerdings nicht, dass Gervasius die Notizen Konrads kannte und benutzte; da aber dieses der Zeit nach möglich und bei der Tendenz der Otia durchaus wahrscheinlich ist, so dürfen wir die Unabhängigkeit der Letzteren in Zweifel ziehen. Mit einer hier nicht in Betracht kommenden Ausnahme macht Gervasius seine Angaben, ohne zu behaupten, dass er das Gegebene selbst gesehen oder gehört habe, aber auch ohne bei seiner Compilation eigene Erinnerungen und Phantasien auszuschliessen. Dass Gervasius ebensowohl als Konrad mit der gelehrten Litteratur und ihren Traditionen vertraut war, versteht sich von selbst.

Nach allem diesem wird es gerechtfertigt erscheinen, wenn wir, im Gegensatz zu Roth und Comparetti, in Anbetracht seines höheren Alters das Werk eines „Nichtaugenzeugen“ zur ersten Beweisaufnahme hinzuziehen, nämlich die Schrift De natura rerum (um I I $85^{2}$ ) von Alexander Neckam, und auch die wenigstens den Otia voraufgehende Chronik Helinands (vor I 204) zur Vergleichung dazunehmen. Ist im Vorhergehenden dargethan, dass weder Konrad noch Gervasius Berichterstatter neapolitanischer Volkssagen sind, noch auch sein wollen, so gibt, weil dieser Prätension fern, Alexanders Buch so wenig wie Helinands Chronik Veranlassung, die darin mitgetheilten Virgillegenden für urpopuläre $z u$ halten. Roths irrthümliche Annahme einer Anwesenheit Alexanders in Neapel und Comparettis Voraussetzung einer von Gervasius und Konrad unabhängigen Verbreitung der Neapelsage haben daher kein entscheidendes Gewicht in der Frage.

Wir sehen nun zu, wie die einzelnen Legenden sich bei Neckam, Konrad, Helinand und Gervasius verhalten und entwickelt haben.

I. Macellum. Alexander Neckam redet, wie von einer bekannten Thatsache, von des „Virgilii prudentia carnem nescio qua vi herbarum conditam in macello recludentis, quae vingentis annis elapsis recentissima et saporis optimi suavitate commendabilis reperta est." Konrad gibt an, dass sich das Fleisch (jetzt noch) sechs Wochen lang erhalte, und schreibt, gleichwie auch Helinand, dem Virgil die Errichtung des Gebäudes zu. Gervasius bleibt, ohne jedoch eine Zeitangabe zu machen, bei der Fassung Neckams. Bei Konrad und Gervasius besteht erklärlicher Weise das Fleischhaus noch (vgl. auch Konrads sechs Wochen).

${ }^{1}$ Ein Pröbchen mag zur Bestätigung hier Platz finden: De faba versa et eius efficacia. Est autem huius potentia satis ridiculosa; si enim ipsam cum oratione dominica ter repetita genu flexo collegeris: orans ut qualem vultum praetendis, talem gustans ex ea protrudas; sine dubio hunc ipsum habere effectum conspicies. Si colligendo ridens, risum ex ea speras, gustans usque ad solis occasum ridebis sine intermissione ... Nec fidem his dedissem, si non ipse probassem. III. D. cap. XIV.

${ }^{2}$ Vgl. Comp. II, 30. Wright, Al. Neckam, S. XIV. 
2. Hortus. Neckams Luftmauer und Luftbrücke konnte Konrad nicht wohl gebrauchen. Auch Gervasius erwähnt sie nicht. Der Garten cnthält nach des Letzteren Bericht medicinische Kräuter. Bei Helinand lesen wir: "Et hortum quemdam sic fecisse dicitur ut in eo non plueret."

3. Serpentes. Durch einen goldnen Blutegel hielt nach Neckams Angabe "der Dichter von Mantua" das Gewürm in den Canälen der Stadt gebannt. „Caeterum ad mentem reducimus, heisst es bei Konrad, quod apud Neapolin est quaedam porta ferrea... in qua Virgilius omnes serpentes illius regionis inclusit." Diese porta ferrea fällt bei Gervasius zusammen mit der ,porta dominica Nolam respiciens . . . in cujus ingressu est via lapidibus artificiose constructa; sub hujus viae sigillo conclusit Virgilius omne genus reptilis nocui:“ Die Abweichungen reichen hin, eine "telestische“ Auffassung unwahrscheinlich zu machen. ${ }^{1}$

4. Equus. Die Geschichte von Virgils ehernem Pferd erzählt nur Konrad: "In eadem civitate est equus aereus magicis incantationibus a Virgilio sic compositus, ut ipso integro permanente nullus equus possit indorsari." Die natürlichste Erklärung ist doch wohl die durch die bekannte, aber hierbei sonst übersehene Stelle der Vita: „Quibus rebus [Medicin und Mathematik] cum ante alios eruditior peritiorque esset, se in urbem contulit statimque magistri stabuli equorum Augusti amicitiam nactus multos variosque morbos incidentes equis curavit." 2

5. Balnea. Diese werden erwähnt von Konrad, Helinand und Gervasius. Die Bäder von Bajae waren von gelehrten Schriftstellern mehrfach als eine Merkwürdigkeit besprochen worden. ${ }^{3}$

6. Musca. Konrad erzählt uns, dass in Neapel eine Thür sei, auf welcher Virgil eine Zaubermücke angebracht hatte. Noch unbestimmter heisst es bei Gervasius (nachdem von einem Refectorium - irgendwo ,in regno Arelatensi“ - die Rede war, ,in quo nulla musca detineri potest"): "Porro in Campania, civitate Neapolitana scimus Virgilium arte mathematica muscam erexisse aeneam" etc.; ähnlich wie schon bei Helinand: „In porta Neapolis Campanie dicitur fecisse muscam eneam quae ommes muscas ab urbe expellebat." Höchst wahrscheinlich beruhen diese Angaben auf der von Johannes von Salisbury erzählten Anekdote, ${ }^{4}$ der ich im Gegensatz zu Comparetti auf Grund der Nichterwähnung einer in Neapel vorhandenen Mücke oder Legende seitens des in der Stadt bekannten Johannes, sowie auf Grund der Geschichtlichkeit der in der Anekdote vorkommenden Namen und allgemeinen Verhältnisse ${ }^{5}$ einen litterarischen Ursprung zuschreiben möchte.

1 Eine ähnliche Geschichte steht schon bei Gregor von Tours; vgl. Liebrecht, l. c. S. 98 .

2 Das eherne Pferd stammt nach Warton aus dem Orient und war zu Konrads Zeit bekannt genug.

${ }^{3}$ Vgl. Du Méril, l. c. S. 436 .

- Policraticus I, 4.

${ }^{5} \mathrm{Zu}$ der Bemerkung Comparettis (II, 34) hierüber vgl. das weiter unten über Caraczolos Chronik Gesagte. Auch ist diese ohnehin 223 Jahre nach dem Policraticus erschienen. 
7. Statua. Die Bildsäule hält nach Konrad eine balista tensa in der Hand, bei Gervasius ist dieselbe in eine tuba verwandelt, was sehr gegen die Existenz der Statue spricht. Ohne Beziehung auf Virgil war der Kern der Sage litterarisch vorhanden. ${ }^{1}$

8. Ampulla. Die Fabel von der Zauberflasche ${ }^{2}$ mit dem Abbild von Neapel (bei Konrad) scheint allerdings eine Anspielung auf den Volksglauben zu enthalten, wenn es von dieser heisst: „in cujus integritate tantam habebant [cives] fiduciam, ut eadem ampulla integra permanente nullum pati posset civitas detrimentum." Hat nun aber auch eine solche Tradition wirklich bestanden, so kann doch der Bezug auf Virgil von Konrad herrühren. Der Dichter galt ja bereits für den Lehnsherrn und nach Konrad auch für den Gründer der Stadt, und es musste dem Berichterstatter ein besonderes Relief verleihen, dem virgilischen Zauber zum Trotz Neapel eingenommen zu haben ${ }^{3}$; freilich (denn die Zauberkraft soll nicht illusorisch erscheinen) "forte quia ampulla modicum fissa est, civitate nocuit."

9. Capita. Die Existenz der von Gervasius erwähnten Steingeschichten scheint anderweitig gesichert. Dass wir es bei Gervasius mit einem wirklichen Erlebniss zu thun haben, beweist mehr als das "quod illic expertus sum" die Ausführlichkeit der Erzählung. Wurden aber auch von dem Archidiakonen von Neapel die Zaubergesichter dem Virgil zugeschrieben, so war jener ja des Gervasius Zuhörer "in jure canonico apud Bononiam, scientia illustris". Die „traditionelle Erzählung“ des Volkes lautete eben anders und wusste nichts von Virgil. 4

Io. Ossa. Die Behauptung Konrads "quod vidimus et probavimus" zeigt deutlich genug, dass die ganze abenteuerliche Geschichte nicht eine Volksansicht wiedergibt. Uebrigens ist der Zusammenhang mit den Sagen über Virgils Grab nicht zu verkennen.

I I. Sepulcrum. Des Gervasius Erzählung, dass ein Engländer in Neapel Virgils Gebeinen nachgeforscht, von den Neapolitanern aber an ihrer Wegführung gehindert worden sei, hängt mit der Notiz des Johannes von Salisbury, wonach ein gewisser Ludovicus solche Zwecke verfolgte, offenbar zusammen. In beiden Fällen ist mit Schaarschmidt 5 und Comparetti das Bestehen der Virgilsage vorauszusetzen. Auch hat ohne Zweifel, wie Comparetti weiter angibt, der Bericht des Johannes einen ursprünglichen, realen, der des Gervasius einen wesentlich legendären Charakter. Dass aber die Kenntniss und Ausschmückung der Sage eine populär-neapolitanische gewesen sei, ist durchaus nicht anzunehmen, und die Verweigerung der Gebeine von Seiten des Volkes beweist nichts gegen dessen

'Scoppa, der 1507 schreibt, spricht von Marmor-Statuen und kann hier nichts beweisen (Comp. II, 35). Vgl. auch Liebrecht, l. c. S. 107.

${ }^{2}$ In späteren Berichten ist es ein verzaubertes $\mathrm{Ei}$.

3 Vgl.: . . ",porta firmissima, instar castelli aedificata valvas habens aeneas, quas nunc tenent satellites imperiales."

- Vgl. Comp. II, 35 und die Anm. zu No. 7.

'In seinem Fohannes Saresberiensis etc. 
von Crervasius gradezu hervorgehobene Unbekanntschaft mit dem (irabe der Dichters; der Cultus, den man in früherer Zeit dem (irabe Virgils gewidmet, war ja auch von litterarischen Kreisen ausgregangen. Ueberdies wird man an der Identität des Zauberbuches bei (iervasius mit dem des Dolopathos etc. kaum zweifeln können.

1 2. Rupes. Vielleicht darf man bei der Mittheilung des Gervasius "De rupe incisa quae nullus admittit insidias" _ "Arte mathematica haec operatus est Virgilius, quod in illo montis opaco inimicus inimico si ponit insidias, nullo dolo nullove fraudis ingenio suae malitiae in nocendo dare potest effectum" 1 - an das bekannte Distichon der Vita denken:

\section{„Monte sub hoc lapidum tegitur Balista sepultus, Nocte die tutum carpe, viator, iter."}

Ich schliesse hieran meine Vermuthungen über. die Entstehung der einzelnen Sagen, die wir uns, wie wir nun wohl behaupten dürfen, im Ganzen nicht als an neapolitanische Kunstobjecte geknüpft, sondern als auf der Vorstellung der Gelehrten von der übernatürlichen Weisheit des Virgil, namentlich in Mathematik und Medicin, beruhend $z \mathfrak{u}$ denken haben.

Bei der Sage von der Statue, welcher ja Konrad eine balista zuschreibt, lässt sich wohl ebenfalls eine Einwirkung des soeben erwähnten Epigrammes annehmen. Der Nachdruck liegt hier wie dort auf mons, balista, tutum. Aehnlich könnte man den Anlass zu dem Umstande, dass Virgil die Verfertigung einer Zauberfliege zugeschrieben wird, in den Worten der Vita "fecit . . . e et culicem" finden. Und wenn es dort heisst ,serpentem interemit", so hätte auch die Schlangenvertilgung ihre classische Stelle. Schliesslich ist vielleicht der Ursprung der macellum-Sage in dem Namen Marcellus zu suchen. Ein Ausdruck wie ,marcellum instruxit" konnte von einem Gelehrten des 12. Jh. recht wohl von der Erbauung eines Fleischhauses verstanden werden. Dass dann Gervasius eine ursprünglichere Fassung der Sage bewahrt hätte, als seine Vorgänger, kann nicht befremden, da ihm die gesammte gelehrte Tradition zu Gebote stand.

Auf die römische Virgillegende von der salvatio Romae brauchen wir nicht weiter einzugehen. Sie ist, wie es ohne die scheinbaren ausdrücklichen Beweise auch mit den ganz ähnlichen neapolitanischen Fabeln der Fall gewesen sein würde, niemals für eine Localtradition angesehen worden. Es sei nur noch bemerkt, dass die ältere römische Sage sich auch noch deutlicher an die Vorstellung von Virgils Prophetie anschliesst. Dies erhellt aus einem Vorgesang zu der Geste des Loherains ${ }^{2}$, der uns zugleich zeigt, in welcher

${ }^{1}$ Konrad spricht von einem ,mons barbarus, ad quem per viam subterraneam per medium montis accessimus", aber ohne den Virgil dabei zu erwähnen. Leibnitz $\Pi, 698$. Liebrecht S. I08.

${ }^{2} \mathrm{Vgl}$. Stengel, Mittheilungen aus frz. Hss. der Turiner Universitäts. Bibliothek. S. $12 \mathrm{ff}$. 
Weise die Vulgärpoesie zu der Bildung des Virgilmythus beigetragen hat. 1

Woher kommt es aber wohl, dass Konrad und Gervasius gerade Neapel zum Schauplatz der virgilischen Wunder machen? Darauf ist Folgendes zu erwiedern. Einmal stand in der Vita zu lesen, dass Virgil nach Rom höchst selten ging, in Neapel dagegen seine mathematischen und medicinischen Studien betrieb und hier auch begraben ward. Ferner hatte ja Konrad nach der Einnahme der Stadt Neapel daselbst seinen Wohnsitz aufgeschlagen, während er mit Rom nicht in nähere Berührung kam. Endlich war nach der Sage der römische Zauberbau bei Christi Geburt zusammengestürzt, folglich konnten Konrad und Gervasius nicht vorgeben, ihn gesehen zu haben.

Eine wichtige Frage ist noch übrig: die nach einheimischen „populären“ Berichten über die neapolitanische Sage vom Zauberer Virgil. Ist es nun schon auffallend genug, dass kein früheres Zeugniss der localen Volkslitteratur sich auffinden lässt als die im Jahr r 382 geschriebene Cronica di Partenope, so liegt in der Beschaffenheit der darin enthaltenen Berichte gradezu ein Beweis für die nicht-locale Entstehung der Tradition. Obgleich Neapolitaner, so bemerkt Comparetti, habe sich Bartolomeo Caraczolo nicht an das allein gehalten, was man in Neapel zu seiner Zeit sich über Virgil erzählt habe. Allein der. Virgil der Cronica di Partenope ist der Hauptsache nach gar nicht der traditionelle, sondern der historische. Er ist der grösste von allen Dichtern, der Verfasser der Georgica, der Eklogen und der Aeneide; er lebt zur Zeit des Octavian und is stirbt in Brundusium, von wo seine Gebeine nach Neapel übergeführt werden. Allerdings berichtet der Chronist auch die über Virgil erzählten Wunderdinge, aber er bemerkt ausdrücklich, dass er aus Alexanders und des Gervasius Schriften geschöpft habe. Wozu dies, wenn er eine lebendige Volkssage mittheilen wollte und an deren Quelle sass? Einige Aenderungen in den Legenden veranlassen Comparetti zu der Bemerkung, Caraczolo müsse, wenn Neckam unter Alexander zu verstehen sei, ein interpolirtes Exemplar der Schrift De natura rerum vor sich gehabt haben. Aus dem Titel Risponsi imperiali könnte man in Bezug auf das Werk des Gervasius auf das Gleiche, vielleicht auf eine italienische Bearbeitung schliessen. Eine französische Uebersetzung der Otia zeugt jedenfalls für deren rasche Verbreitung. ${ }^{2}$ Hätte also auch, zweihundert Jahre nach dem Erscheinen von Gervasius' Buch, Caraczolo die Virgilsagen nach-

${ }^{1}$ Die Ausbreitung und Erweiterung der Virgilsage durch volksthümliche Schriften, namentlich in Frankreich und Deutschland, zu verfolgen, auch darauf dürfen wir nach Comparettis ausfuhrlicher Darlcgung hier verzichten. Der Zusammenhang mit der gelehrten Litteratur tritt übrigens, trotz der allmählichen Wandlung des unschuldigen Zauberers zum Schwarzkünstler und Wüstling, selbst in neuen Zügen noch hervor.

2 Vgl. Liebrecht S. VII. 
weislich im neapolitanischen Volke gesammelt, so folgte daraus noch gar nichts für ihre locale Entstehung. Ausserdem enthält Caraczolos Relation über die salvatio Romae einen Zug, der erst in den französischen Romanen des 13. Jh. zum Vorschein kommt. ${ }^{1}$ Dass er noch andere als die genannten Quellen vor sich gehabt, erklärt der Chronist ausdrücklich, und wenn er ihren Angaben weniger Glauben schenkt, so macht dies nicht allein „seinem italienischen gesunden Menschenverstand Ehre", sondern zeigt, dass sein eigner Bericht, anstatt den gleichzeitigen Volksglauben wiederzuspiegeln, eine Auswahl schriftlich verbreiteter Sagen ist. Obwohl weniger kritisch als der Chronist von Neapel, hat auch der Mantuaner Bonamente Aliprando durchaus litterarische Quellen benutzt. Auch er verliert niemals den Dichter Virgil aus den Augen. Keine italienische Stimme legt für den volksthümlichen Ursprung virgilischer Sagen Zeugniss ab. Antonio Pucci kennt die Tradition, aber auch die französischen Romane. Im allgemeinen aber, das gesteht Comparetti selbst $\mathrm{zu}$, scheint die Virgilsage in Italien am wenigsten Anklang gefunden $z u$ haben. Aliprando selbst ist "mit dem grössten Theil der neapolitanischen wie der ausländischen Legende unbekannt", und Boccaccio, "der Neapel sehr wohl kennt", "weiss von nur zwei oder drei Thatsachen" der Tradition von Neapel (Comp. II, I 29. 142). Nur im Anfang unseres Jahrhunderts soll, nach der Versicherung einiger Reisenden, die Sage von dem Zauberer Virgil sich unter dem neapolitanischen Volke gezeigt haben. Dort war ihres Bleibens nicht. Lange aber wird sie noch fortleben auf dem heimischen Boden der Litteratur. ${ }^{2}$

${ }^{1}$ Vgl. Comp. II, I 80. I 88.

2 Ein neuer Beweis für das Interesse, welches Prof. Comparettis Werk für die Virgilsage wachgerufen hat, ist G. Boissiers Aufsatz im 3. Heft der Revue des deux mondes 1877 . Bei vorstehender Untersuchung, welche im Wesentlichen übrigens schon 1874 zum Abschluss kam, konnte jener Artikel nicht mehr berücksichtigt werden, brauchte es aber auch nicht, da Boissier sich auf eine lobende Analyse des Virgilio nel medio evo beschränkt hat.

WILHELM VIETOR. 